\title{
A simple model for thermodynamic mixing properties of solid solutions with long- and short-range order
}

\author{
Xin LiU ${ }^{1 *}$, Victor L. VinOGRAD ${ }^{2 *}$, SERGII NichenKO 3 , \\ DMITRII A. KULIK ${ }^{3}$, XIANCAI LU ${ }^{1}$, BJÖRN WINKLER ${ }^{4}$
}

${ }^{1}$ State Key Lab for Mineral Deposits Research, School of Earth Sciences and Engineering, Nanjing University, Xianlin Avenue 163, 210023 Nanjing, China

(*correspondence:xinliu.nju@gmail.com)

${ }^{2}$ Institute of Energy and Climate Research (IEK-6),

Forschungszentrum Jülich, Wilhelm-Johnen-Straße, 52425 Jülich, Germany

${ }^{3}$ Paul Scherrer Institut, Nuclear Energy and Safety Division, 5232 Villigen PSI, Switzerland

${ }^{4}$ Institute of Geosciences, Goethe Universität Frankfurt am Main, Altenhöferalle 1, 60438 Frankfurt am Main, Germany

Compound Energy Formalism (CEF) for solid solutions is widely used in thermodynamic assessment studies of multicomponent systems. However, it typically overestimates order/disorder transition temperatueres by not taking into account short-range ordering (SRO) in nominally disordered phases. Using calcite-magnesite-dolomite system as an example we show that the effect of SRO can be emulated within the CEF by including an extra negative excess enthalpy term causing an additional long-range ordering (LRO) effect over an intermediate temperature range. The mathematical form of the extra term ensures that its effect vanishes both in low- and high-temperature limits imitating the vanishing of SRO in the same limits. The modified CEF model (mCEF) that includs such a term significantly improves the fit to phase relations in the calcte-dolomitemagnesite-dolomite system. Another advantange of the mCEF is that it needs only few fitting parameters. Alternatively, their values can be obtained by perfoming first principles based calculations on supercell structures containing single subsitutional defects. This offers a good balance between accuracy and computational costs. The obtained free energy equations can be used within a wide variety of software tools dedicated for phase equilibrium calculations. 